\title{
Zwei-Stufen-Theorie und praktische Überlegung
}

\author{
Anna Kusser
}

In seinem einflußreichen Aufsatz mit dem Titel „Freedom of the Will and the Concept of a Person" hat Harry Frankfurt die These vertreten, daß sich Personen von Tieren und anderen nicht-personalen Wesen dadurch unterscheiden, daß sie zu ihren eigenen Wünschen Stellung nehmen.

Die Idee, es zeichne den Menschen aus, daß er auf Distanz gehen könne zu seinem Wollen, seinen Wünschen und Begierden ist weit verbreitet. Vernunft gilt dabei als Vermögen der Überlegung und als Ort von umfassendem Wissen, als ein Vermögen mithin, das aus dieser Distanz heraus das Wollen einer Prüfung unterwerfen kann. Frankfurts Menschenbild steht in dieser Tradition, nimmt aber auch in bedeutsamer Weise von ihr Abstand. Denn bei Frankfurt ist es nicht die Vernunft, sondern wiederum der Wille, von dem her die Stellungnahme zum eigenen Wollen und Wünschen erfolgt. Der Wille gibt also gewissermaßen die Prüfungsinstanz für sich selber. Das erfordert eine interne Differenzierung in mindestens zwei Instanzen, woraus eine reflexive und gleichzeitig hierarchische Willensstruktur resultiert. Diese Struktur, so meint Frankfurt, stellt das spezifische Kennzeichen von Wesen dar, die Personen sind; denn diese Struktur ist bei einer bloß willentlichen Handlungsteuerung, die man auch schon bei Tieren findet, noch nicht gegeben.

Im Anschluß an diesen Personenbegriff faßt Frankfurt dann Freiheit oder Autonomie als ein geglücktes Personsein auf. Freiheit oder Autonomie sollen nämlich dann gegeben sein, wenn eine willentliche Stellungnahme höherer Stufe nicht bloß gegeben ist, sondern praktisch bestimmend und wirksam wird, d. h. im Handeln durchschlägt.

Die von Frankfurt entworfene reflexive Struktur von Personalität und Freiheit ist von beträchtlicher intellektueller Anzichungskraft, wovon nicht zuletzt der vorliegende Band zeugt. Diese Auffassung des menschlichen Willens ist vielfach aufgegriffen und von angrenzenden Disziplinen als brauchbar wahrgenommen worden. ${ }^{1}$

Nichtsdestotrotz möchte ich hier die These vertreten, daß die zweistufige Willenstheorie eine Konstruktion im schlechten Sinne darstellt. Sie verzeichnet die von ihr unmittelbar intendierten Phänomene und impliziert gleichzeitig eine Auffassung ratio-

1 So etwa in der Moralpsychologie; vgl. Nunner-Winkler (1993) und (2000). 
nalen Handelns, die inadäquat ist, weil sie das entscheidende Element praktischer Überlegung ausblendet.

Den unmittelbaren Anknüpfungspunkt meiner Auseinandersetzung mit Frankfurt bilden nicht seine Thesen über Personalität und Willensfreiheit, sondern die Phänomene mit Bezug auf die er die zweistufige Willensauffassung einführt. Diesen Phänomenen gebührt insofern besondere Aufmerksamkeit, als sich an ihnen erweisen soll, daß die Zwei-Stufen-Struktur des Willens kein leeres theoretisches Hirngespinst, sondern psychologisch real und empirisch fruchtbar ist.

Ich möchte zunächst die innere Logik von Frankfurts zweistufiger Konzeption an Hand der zentralen Phänomene nachzeichnen. Auf diese Weise soll ein detailliertes und zunächst möglichst überzeugendes Bild dieser Konzeption entworfen werden.

Im Anschluß daran werde ich einen Einwand gegen diese Sicht nach verschiedenen Seiten hin entfalten. Am Ende wird kurz ein alternatives Bild entworfen, das wichtige Motive bei Frankfurt aufnimmt, gleichzeitig aber die praktische Überlegung in ihr Recht setzt.

\section{Drei Unterscheidungen}

Begrifflich gesehen bilden drei Unterscheidungen das Grundgerüst der Zwei-StufenKonzeption; die erste ist die Unterscheidung zwischen Wünschen und Wollen.

Wünsche oder, vorerst eingeschränkt, Wünsche erster Stufe, sind ,simply desires to do or not to do one thing or another." ${ }^{\prime \prime}$ Der Begrilli des Wunsches sei, so Frankfurt „extraordinarily elusive"; er unternimmt es nicht, diesen Begriff genauer zu definieren, sondern gibt stattdessen eine Reihe von Sätzen an, die mit der Feststellung, eine Person, wünsche etwas, verträglich sind. Zum Beispiel könne man etwas wünschen, ohne daß der Gedanke daran einen positive Emotion auslöst, man könne etwas wünschen, ohne es zu wissen, oder auch ohne es ,wirklich“ zu wünschen.

Frankfurt umschifft mit diesem großenteils unerläuterten Wunschbegriff die Untiefen notorischer Diskussionen rund um den Wunschbegriff, wie z.B. die, ob Wünsche verspürt werden, ob es unbewußte Wünsche gäbe, und wie man überhaupt Wünsche genauer zu fassen habe, etc. Daß Frankfurt diese Gewässer zu umschiffen wünscht, ist nachvollziehbar genug, eröffnen doch die genannten Fragen ein außerordentlich weites Feld. ${ }^{2}$ Aber in Frankfurts Abstinenz gegenüber dem Wunschbegriff steckt auch eine weiterreichende Annahme, die ich explizit hervorheben möchte: Es ist die Überzeugung, daß von der Beantwortung dieser Fragen bzw. einer genaueren Fassung des Wunschbegriffs für seine Konzeption nichts Entscheidendes abhängt.

Hingegen ist der Zusammenhang zwischen Wünschen und Handlungen außerordentlich wichtig für Frankfurts Konzeption. Zunächst hält er fest, daß Wünsche nicht unbedingt zu entsprechendem Handeln führen. Im Anschluß daran differenziert er dann den Wunschbegriff: Wünscht eine Person etwas und handelt dann auch entsprechend, dann ist von einem Wollen sprechen. Eine Person kann demnach vieles wünschen, doch will

l Frankfurt (1971), 7.

2 Vgl, Kusser (1989), Teil II. 
sie jeweils blol3 das. was sie dam auch tut. Der Begritt des Wunsches ist also der allgemeine Begriff, während der Begriff des Willens eine Sperialisierung derart ist, daß der Wille ciner Person der jeweils handlungseffektive Wunsch ist.

Soweit die wichtigen Begriffe erster Stufe; mit einer weiteren Unterscheidung wird dann die zweite Stufe eingeführt. Von Wünschen zweiter Stufe will Frankfurt sprechen, wenn sich Wünsche auf Wünsche beziehen; sie also Wünsche erster Stufe zum Gegenstand haben. Die zweite Stufe bringt nun das reflexive Moment. Im Prinzip könnte über der zweiten Stufe nach demselben Konstruktionsprinzip eine dritte errichtet werden, auf der sich Wünsche auf Wünsche zweiter Stufe beziehen, usw. Frankfurt arbeitet jedoch nur mit zwei Stufen. ${ }^{1}$

Auf der zweiten Stufe ist die dritte wichtige Unterscheidung angesiedelt, die Unterscheidung zwischen Wünschen und Volitionen zweiter Stufe. Diese Unterscheidung ist eine Binnenunterscheidung der zweiten Stufe und das Kriterium betrifft den Gehalt. Wünsche zweiter Stufe beziehen sich darauf, Wünsche erster Stufe bloß zu haben. Ein solcher Wunsch unterstellt, daß man den gewünschten Wunsch erster Stufe nicht hat sonst hätte es ja keinen Sinn ihn zu wünschen. Wer einen solchen Wunsch zweiter Stufe hat, der möchte offenbar "schmecken” oder erleben, wie es ist, einen bestimmten Wunsch (erster Stufe) zu haben. So könnte z.B. eine Frau den Wunsch haben, zu erleben, wie es ist, eine andere Frau zu begehren. Sie würde dann etwas über ihre eigene wünschende Verfassung wünschen, sie hätte einen Wunsch zweiter Stufe. Frankfurt hält solche Wünsche 2. Stufe für relativ marginale Erscheinungen. ${ }^{2}$ Sie spielen in seiner Konzeption keine wichtige Rolle, er verwendet sie nur als Gegenstück zu den sogenannten Volitionen zweiter Stufe, denen dagegen eine tragende Rolle zukommt.

Wünscht man von einem bereits auf erster Stufe gegebenen Wunsch, er solle der effektive Wunsch sein, so spricht Frankfurt von einer Volition zweiter Stufe oder einfach einer Volition. Vollständig formuliert hat eine Volition die Form: Ich wünsche, daß mein Wunsch, daß X der Fall ist, der effektive sein möge. Volitionen zweiter Stufe setzen an bereits vorhanden Wünschen erster Stufe an. Man wünscht in ihnen, daß gewisse schon vorhandene Wünsche eine bestimmte Verfassung haben mögen, nämlich die, wirksam zu sein und zum Handeln zu führen.

Die Unterscheidung zwischen Wünschen und Volitionen zweiter Stufe ist nicht parallel zur Unterscheidung zwischen Wünschen und Wollen erster Stufe. Wünschen und Wollen erster Stufe können denselben Gehalt haben, sie unterscheiden sich allein durch die Effektivität. Der sprachlichen Formulierung kann man es daher nicht ansehen, ob eine Einstellung erster Stufe ein Wunsch oder ein Wille ist. Selbst wenn jemand entschlossen bekunden würde, er wünsche $\mathrm{X}$ nicht bloß, sondern wolle es, so kann sich die Richtigkeit dessen, Frankfurt folgend, erst im Handeln zeigen.

Dagegen ist die Unterscheidung von Wünschen und Volitionen zweiter Stufe bereits in der Formulierung greifbar, weil sie den Gehalt betrifft. Im einen Fall wünscht man eine Wunsch zu haben, im anderen wünscht man, ein Wunsch sei effektiv. Eine Volition zweiter Stufe operiert demnach wie ein Zeiger, der über der Menge vorhandener

\footnotetext{
Kritiker haben hier einen unbegrïndeten Abbruch bzw. die Gefahr eines unendlichen Regresses gesehen. Vgl. Wolf (1990), Gosepath (1992).

: II. Steinfath widerspricht Frankfurt in diesem Punkt, vgl. den Beitrag in diesem Band.
} 
Wünsche erster Stufe schwebt, dann auf einen Wunsch zeigt und damit besagt: Dieser da soll der effektive Wunsch sein! Die Volition selegiert einen Wunsch erster Stufe und designiert ihn zum Willen.

Volitionen zweiter Stufe sind auf diese Weise auf ein Wollen erster Stufe gerichtet, sie sind aber nicht genau parallel konstruiert. Der Parallelbegriff zum Wollen erster Stufe ist der einer effektiven Volition zweiter Stufe. Frankfurt führt dafür keine eigene Bezeichnung ein, obzwar dieser Begriff bei ihm eine wichtige Rolle spielt, da er den Kern seiner Auffassung von Freiheit ausmacht. Ich möchte von durchschlagender Volition sprechen. Eine durchschlagende Volition würde tatsächlich zu dem in ihr Gewünschten - nämlich dazu, daß ein Wunsch (1. Stufe) effektiv sei - führen. Sie würde sozusagen, wie der Wille auf der ersten Stufe, für ihre eigene Erfüllung sorgen. Wünsche ich, mich an der Nase zu fassen und tue es dann aufgrund dessen, so hat dieser Handlungswunsch erster Stufe seine eigene Erfüllung in Form einer Handlung verursacht. Ebenso erfüllen sich durchschlagende Volitionen zweiter Stufe selbst, indem sie das in ihnen Gewünschte verursachen. Denn wenn Volitionen 2. Stufe durchschlagen, verwandeln sie bloße Wünsche 1. Stufe in ein Wollen. Und weil dieses Wollen erster Stufe dann per definitionem zum Handeln führt, schlagen effektive Volitionen 2. Stufe indirekt aufs Handeln durch.

\section{Die Konzeption der Zentralphänomene}

Volition und durchschlagende Volition zweiter Stufe sind komplexe Begriffe; man durchschaut sie nicht sogleich, sondern muß sie sich erst klar machen. Die Wünsche, die sie bezeichnen sollen, sind ebenfalls komplexe psychische Entitäten, die man vermutlich ebenfalls nicht spontan auffasst. Es dürfte nicht leicht sein, den eigenen Wunsch, spazieren zu gehen - ein Wunsch erster Stufe - und den Wunsch, den Wunsch zu haben, spazieren zu gehen - eine Wunsch zweiter Stufe - auseinander zu halten, und von beidem wiederum die Volition zweiter Stufe, der Wunsch spazieren zu gehen, möge effektiv sein, zu unterscheiden.

Nun soll aber andererseits vom Vorhandensein von Wünschen und Volitionen zweiter Stufe viel abhängen, nämlich ob ein Wesen den Status einer Person hat. Daher sollte die Frage, unter welchen Bedingungen man nicht umhin kann, einem Wesen eine zweistufigen Willen zuschreiben, eine überzeugende Antwort erhalten.

Die bewußtseinsmäßige Evidenz höherstufiger Wünsche aus der Perspektive der ersten Person ist für Frankfurt generell kein Thema, was eine beachtenswerte Lücke darstellt. Er führt den Aufweis der „Realität“ höherstufiger Wünsche nicht über ihre bewußtseinsmäßige Gegebenheit, sondern rein theoretisch-konzeptionell. Es gibt Phänomene, so meint er, die wir nur zureichend beschreiben können, wenn Zweistufigkeit eingeführt wird. Diese Zentralphänomene bilden den primären empirischen Referenzpunkt der Zwei-Stufen-Theorie. In einem zweiten Schritt wird diese dann ausgeweitet auf alles Handeln von Personen.

Es ist der erste und entscheidende Schritt dieses Vorgehens - die Anwendung der Zwei-Stufen-Konzeption auf die Zentralfälle -, die mich hier vornehmlich beschäftigen wird. Meine These wird sein, daß die Zwei-Stufen-Theorie bereits diese Fälle nicht 
adäquat beschreibt. Es handelt sich um Fälle, da jemand mit seinem Handeln nicht einverstanden ist, gleichzeitig dieses Tun aber nicht kraß unwillentlich, sondern aus eigenem Antrieb geschieht. Frankfurts Beispiel ist das eines Drogensüchtigen, der immer wieder zur Droge greift. Frankfurt schildert ihn so:

"One of the addiets hates his addiction and always struggles desperately, although to no avail, against its thrust. He tries everything that he thinks might enable him to overcome his desire for the drug. But these desires are too powerful for him to withstand, and invariably, in the end, they conquer him. He is an unwilling addict, helplessly violated by his own desires."

Frankfurt konzipiert diesen Fall eines Drogensüchtigen „wider Willen“ folgendermaBen: Der Süchtige hat konfligierende Wünsche erster Stufe: Er 'wünscht die Droge zu nehmen und er wünscht gleichzeitig, sie nicht zu nchmen. Darüber hinaus hat er noch eine Volition zweiter Stufe. Durch sie wünscht er, der Wunsch, die Droge nicht zu nehmen, sei derjenige, der sein Handeln bestimmt. Er wünscht damit auf zweiter Stufe, dieser Wunsch möge nicht bloß eincr seiner Wünsche, sondern der effektive Wunsch, d.h. sein Wille sein.

Faktisch ist das nicht der Fall, denn er bleibt nicht abstinent. Der andere Wunsch, der Wunsch, die Droge zu nehmen, ist entgegen seiner Volition zweiter Stufe sein Wille. Der Süchtige hat also nicht den Willen, den er auf zweiter Stufe zu haben wünscht. Zwischen seiner Volition zweiter Stufe und seinem Willen besteht Dissonanz. Was er auf zweiter Stufe wünscht, wird von der ersten Stufe, an die sich der Wunsch richtet, nicht erfüllt.

Frankfurt kontrastiert den Süchtigen wider Willen mit einem anderen Süchtigen, den er einen „wanton“" nennt. „Wanton" bedeutet in etwa Lüstling, zügelloser Mensch. Ich möchte im folgenden vom haltlos Drogensüchtigen sprechen. Er wird von Frankfurt so beschrieben:

"His actions reflect the economy of his first-order-desires, without his being concerned whether the desires that move him to act are the desires by which he wants to be moved to act. If he encounters problems in obtaining the drug or in administering it to himself, his responses to his urges to take it may involve deliheration. But it never oceurs to him to consider whether he wants the relation among his desires to result in his having the will he has."

Der entscheidende Unterschied zwischen dem haltlos Drogensüchtigen und dem Drogensüchtigen wider Willen soll nun der sein, dal3 der haltlose keine Wünsche zweiter Stufe hat. Beide haben auf der ersten Stufe konfligierende Wünsche - sie wollen die Droge nehmen und sie wollen sie nicht nehmen - und bei beiden ist der Wunsch sie zu nehmen der stärkere - er ist ihr Wille. Nur soll der haltlos Drogensüchtige dazu quasi keine Meinung haben. Es ist ihm egal bzw, jederzeit recht, wo ihn seine Wünsche hinführen und wie er handelt. Der Drogensüchtige wider Willen dagegen wünscht es sich anders als es geschieht; er wollte, er würde anders handeln. Das heißt, er wünscht auf zweiter Stufe, dies möge nicht sein Wille sein. Soweit die zweistufige Konzeption der B̈eiden Beispiele.

1 Frankfurt (1971), 12.

2 Ebd. 
Um diese Sicht zu vertiefen, möchte ich in einigen Punkten ihrer inneren Logik weiter nachgehen. Zunächst sei betrachtet, wie hier die Verhältnisse auf der ersten Stufe dargestellt werden. Es soll zunächst ein Konflikt zweier gegensätzlicher Wünsche herrschen: dem Wunsch, die Droge zu nehmen und dem Wunsch, die Droge nicht zu nehmen. Außerdem soll der Wunsch, die Droge zu nehmen, der effektive Wille des Süchtigen sein. Denn $\mathrm{es}$ ist dieser Wunsch, der sein Handeln bestimmt. Damit sind die bereitgestellten Begrilfe erster Stufe ausgeschöpft. die Beschreibung der Verhältnisse auf erster Stufe ist abgeschlossen.

Aus dem Beispiel geht nun aber klar hervor, daß der Drogensüchtige die Drogen irgendwie gegen seinen Willen nimmt. Er selbst findet seinen Drogenkonsum problematisch und lehnt ihn ab. Dieser wichtige Zug des Beispiels ist noch nicht ins Bild gebracht; denn die Feststellung, es liege ein Wunschkonflikt vor, reicht dafür nicht aus. Sicher, dieser Süchtige wünscht einesteils, die Droge nicht zu nehmen. Dem steht aber auf der anderen Seite sein Wunsch nach Drogenkonsum entgegen, der offenkundig stärker ist.

Die Beschreibung der Verhältnisse erster Stufe - zwei Wünsche, einer davon der stärkere und der Wille - spricht somit auf das subjektive Problem des Drogensüchtigen überhaupt noch nicht an. Das heißt umgekehrt, daß die Zwei-Stufen-Theorie eine praktisch gesehen problemfreie Beschreibung der Verhältnisse auf erster Stufe gibt. Das Moment der Unwillentlichkeit bleibt auf der ersten Stufe, so daß der Eindruck entsteht, als sei die zweite Stufe erforderlich, um genau diesen Punkt zu artikulieren.

Der haltlos Süchtige illustriert als Figur, daß das Problem unwillentlichen Handelns auf der ersten Stufe nicht greifbar ist; diese Figur kann als sozusagen lebender Beweis dafür gedacht werden. Wichtig ist hierbei, wie Frankfurt ausdrücklich hervorhebt, daß der Haltlose deshalb nicht irrational ist.

"Nothing in the concept of a wanton implies that he cannot deliberate concerning how to do what he wants to do. What distinguishes the rational wanton from other rational agents is that he is not concerned with the desirability of his desires themselves. He ignores the question of what his will is to be. Not only does he pursue whatever course of actions he is most strongly inclined to pursue, but he does not care which of his inclinations is the strongest. "I

Ausgehend davon, daß das Problem des Drogensüchtigen wider Willen nicht in den Verhältnissen auf erster Stufe liegen kann, erfolgt nun der „Aufstieg” auf die zweite Stufe. Der Drogensüchtige wider Willen, so sieht es Frankfurt, nimmt zu den Verhältnissen auf erster Stufe eine Haltung ein: Er findet, sie seien nicht richtig so, er erklärt sich quasi mit dem Treiben seiner Wünsche nicht einverstanden. Dem haltlos Drogensüchtigen mangelt genau diese zusätzlich Einstellung. Er ist Spielball seiner Wünsche, weshalb er mit seinem Drogenkonsum keine Probleme hat.

Das Zwei-Stufen-Szenarium ist damit komplett: Beide Drogensüchtige haben, erstens, den Wunsch nach Drogenkonsum und den Wunsch nach Drogenabstinenz; bei beiden ist, zweitens, der erste Wunsch der stärkere. Doch findet der unwillentlich Drogensüchtige außerdem noch, daß es genau umgekehrt sein sollte. Er hat damit, drittens,

1 Frankfurt (1971), 11. 
eine Volition zweiter Stufe: Er wünscht, sein Wunsch nach Abstinenz möge der effehtive, der stärkere Wunsch sein.

Das Beispiel der zwei Drogensüchtigen liefert zweifellos eine suggestive lllustration der Zwei-Stufen-Theorie. Es scheint zu zeigen, daß Wünsche zweiter Stufe unumgänglich sind, um Phänomene unwillentlichen Handelns adäqual zu beschreiben. Die hier in Zeitlupe nachvollzogene Rekonstruktion dieses Ansatzes dürfte seine Stärken in helles Licht gerückt haben.

\section{Der Wunschkonflikt}

Im folgenden will ich mich den Schwächen zuwenden. Schon das Resultat des ersten Konstruktionsschrittes, der Wunschkonfliki, ist nicht in der unterstellten Weise gegeben, sondern verdankt sich einer Ausiassung.

Angenomınen, man würde den Drogensüchtigen wider Willen fragen: Willst Du die Droge nehmen oder willst Du sie nicht nehmen? (..Wollen" hier im umgangssprachlichen Sinn verstanden, nicht in Frankfurts terminologischem, also nicht notwendig als effektives Wünschen) Man würde damit nach seinen Wünschen erster Stufe nach „weltgewandten" Wünschen - fragen, denn die Drogen zu nehmen oder nicht zu nehmen, sind äußerer Sachverhalt und nicht wiederum eigene Wünsche.

Er würde sicher nicht antworten, er sei in seinem Wollen hin und her gerissen, weil er beides gleichermaßen wünsche und einfach nicht sagen könne, was er mehr wünsche. Demn dann wäre er in der Lage von Buridans Esel, dem zwei Heubündel gleich verlokkend erscheinen, und der nun nicht weiß, an welchem er knabbern solle. Für den Esel sind beide Alternativen gleich gut, für ihn kommt es nur darauf an, sich iberhaupt nach einer Seite zu wenden.

Wenn dies nicht die Art von Problem ist, die der unwillentlich Drogensüchtige erlebt, dann ist davon auszugehen, daß sich ihın eine der beiden Alternativen bereits auf der ersten Stufe als die Bessere darstellt. Welche könnte das sein? Wäre es der Drogenkonsum, dann wäre das Element der Widerwilligkeił nicht möglich. Denn dann wäre er ein bewußter, überzeugter Drogenkonsument. Also wird sich ihm die Drogenabstinenz als die bessere Möglichkeit darstellen. Wenn dies richtig ist, so herrscht auf erster Stufe kein Konflikt.

Zwar schließen sich beide Alternativen aus und werden auch als solche in Betracht gezogen, doch die entsprechenden Wünsehe ,widersprechen” sich nicht, da sie einen unterschiedlichen Grad haben. Auf Grund der Graduierung herrscht eine präferenzielle Rangfolge zwischen den beiden Wünschen und kein Konflikt.

Trotzdem hat die Vorstellung eines Konflikts etwas Einleuchtendes. Woran liegt dies? Es besteht weniger in einem simultanen Hin- und Hergerissensein, als vielmehr einer inneren Uneinigkeit im Sinne einer mangeinden Konsequenz. Es ist ein Konflikt der primär dadurch beizulegen wäre, daß der Süchtige tatsächlich abstinent bleiben würde. Außerdem wäre von vorneherein diese Art von Konflikt nicht vorhanden, wenn Präferenz und effektive Handlung beim Drogenkonsum lägen. Der gemeinte Konflikt würde sich also immer dann auflösen, wenn Präferenz und Handlung übereinstimmen. 
Der Konflikt, den der unwillentlich Drogensüchtige erlebt, hängt demnach daran, daß er es vorzieht, abstinent zu bleiben, die Droge aber doch immer wieder nimmt. Dies ist ein Konflikt oder besser gesagt eine Unstimmigkeit von Wünschen und Handlungen und kein innerer Konflikt zwischen gegensätzlichen Wünschen.

Die Konstruktion eine Konfliktes auf der ersten Stufe beruht demnach darauf, daß die Graduiertheit von Wünschen ausgeblendet wird. Zieht man sie in Betracht, so bleiben nur zwei Möglichkeiten: Entweder es handelt sich um einen Buridanfall, wo sich zwei gleich gute Alternativen bzw. gleichgewichtige Wünsche gegenüberstehen; das aber wäre nicht der Konflikt des Drogensüchtigen. Oder aber einer der beiden Wünsche prävaliert, dann betrifft der Konflikt ein gestörtes Konsequenzverhältnis von Wünschen und Handlungen; dies ist die Unstimmigkeit, die der Drogensüchtige erlebt.

\section{Ausblendung von Rationalität und Überlegung}

Obgleich pro forma Rationalität und Überlegung auf erster Stufe zugestanden werden, fallen bei der Zwei-Stufen-Konzeption Rationalität und praktische Überlegung unter den Tisch. Rationalität beinhaltet, daß ausgehend von Wünschen und Überzeugungen überlegt wird, welches die rational zu vollziehende Handlung ist. Damit wird zugleich festgelegt, welcher der auf Handlungen gerichteten Wünsche rational gesehen der effektive sein solle. Diese Überlegungstruktur gilt üblicherweise für Wünsche erster Stufe; selbstbezügliche Elemente sind kein wesentliches Element der Struktur.

Hingegen stellt es die Zwei-Stufen-Theorie so dar, als ob der Drogensüchtige wider Willen, solange er nur seine Wünsche erster Stufe in Betracht zieht, keine begründete Ansicht dazu haben könnte, welcher Wunsch effektiv sein solle. Denn - wie oben schon erläutert - die erste Stufe soll nicht genug Substanz haben, um dem Handelnden schon Halt zu geben.

Der praktische Halt, die Handlungsdirektive, ergibt sich für die Zwei-Stufen-Theorie erst daraus, daß von oben her eine Art Zeiger auf einen bestimmten Wunsch deutet der Zeiger ist die Volition - und ihm bedeutet, er solle der effektive sein. Der Handelnde muß, um im Bild zu bleiben, nach oben auf diesen Zeiger blicken, um gewahr zu werden, wie zu handeln wäre. Die handlungsselektive Funktion ist auf der zweiten Stufe angesiedelt. Daher bleibt der Handelnde ohne zweite Stufe bzw. ohne deren Konsultation desorientiert und dem Gerangel seiner Wünsche ausgeliefert.

Tatsächlich bezieht der Drogensüchtige jedoch seinen Halt - seine Meinung, wie es laufen soll - nicht erst aus der Volition zweiter Stufe. Schon auf erster Stufe kommt seine praktische Überlegung zu einem definitivem Ergebnis.' Dieses Ergebnis lautet entweder: Ich will die Droge nehmen - alles in allem; oder aber: Ich will die Droge nicht nehmen, wenn ich all meine Wünsche und die zu erwartenden Folgen bedenke.

Kommt er zu dem zweiten Ergebnis und nimmt dann doch wieder Drogen, so ist er ein Drogensüchtiger wider Willen. Kommt er jedoch zu dem Ergebnis, er wolle sie

Es sei denn, es läge ein Buridan-Fall vor, was aber bereits ausgeschlossen wurde. 
nehmen, so nimmt er die Droge aus Überzeugung und entspricht damit nicht dem Bild eines haltlos Drogensüchtigen.

Die Figur des haltlos Drogensüchtigen stellt überhaupt ein widersprüchliches Zerrbild dar. Er soll einerseits praktisch rational sein und überlegen, andererseits soll es ihm egal sein, welcher seiner Wünsche erster Stufe sich durchsetzt, weil er ja keine Volitionen zweiter Stufe hat: „It never occurs to him to consider whether he wants the relation among his desires to result in his having the will he has." "1

Demnach müßte es dem Haltlosen gleichgültig sein, welche Handlung er vollzieht. Dem widerspricht jedoch, daß er rational überlegt, welche der möglichen Handlungen ein Maximum von Wunscherfüllung verspricht. Auf dieser Handlung wird sich sein konklusiver Wunsch rationalerweise richten. Es wird dem scheinbar Haltlosen daher nicht gleichgültig sein, ob er dieser Handlung vollzieht, bzw. welcher Wunsch der effektive ist.

Die Zwei-Stufen-Theorie erweckt den Eindruck, als müsse jemand, der keine Volitionen zweiter Stufe habe, mit seinen Handlungen stets im Einklang stehen, da sie doch jeweils die Handlungen sind, die er am stärksten wünscht. Richtig ist, das für ein Wesen, das der praktischen Überlegungen fähig ist, die Stärke seiner Wünsche eine wichtige Rolle spielt; denn nur dann kann deliberativ abgewogen und eine praktische Überlegung geführt werden. Doch kann Wunschstärke im Rahmen einer prospektiven Überlegung nicht primär bedeuten, daß von zwei Wünschen derjenige der stärkere ist, der sich im Handeln durchsetzt. Denn für den Überlegenden steht ja gerade in Frage, wie zu handeln sei. Selbst wenn er im voraus wüßte oder annähme, welcher Wunsch in diesem Sinne der stärkere wäre, so könnte er auf der Basis dessen bloß voraussagen, wie er handeln werde. Er könnte jedoch nicht überlegen und festlegen, wie zu handeln sei. Im Rahmen der praktischen Überlegung muß daher Wunschstärke einen anderen Sinn haben als bloße Durchsetzungskraft. Wunschstärke muß den Sinn einer sub-jektiven Bewertung, eines präferentiellen Vorzugs haben. Dann aber ist es kein Auto-matismus mehr, daß ein rational überlegendes Wesen stets so handelt, wie es der - präferentiellen - Stärke seiner Wünsche entspricht. Diese Automatismus gilt nur für Wunschstärke im Sinne von handlungslässiger Durchsetzung.

Der aufgebaute Kontrast zwischen dem haltlos Drogensüchtigen und Drogensüchtigen wider Willen erweist sich damit als unstimmig bzw. fadenscheinig. Wenn der Haltlose, wie Frankfurt annimmt, dieselben Wünsche erster Stufe hat wie der Süchtige wider Willen, so wird sich auch für ihn ergeben, daß es am besten ist für ihn, die Droge nicht mehr zu nehmen. Und wenn er dann trotzdem Drogen konsumiert, so ist auch er ein Süchtiger wider Willen. Auch er wird fühlen, er sei ,helplessly violated by his own desires" - wobei allerdings diese ihn überwältigenden Wünsche nicht identisch mit den in die praktische Überlegung eingehenden präferentiellen Wünschen sind.

An dieser Stelle möchte ich noch einmal zurückkommen auf Frankfurts anfängliche Einführung eines ziemlich vagen Wunschbegriffs. Weder wird dort festgehalten, daß Wünsche stets einen bestimmten Grad haben, noch wird ihre Rolle in der praktischen Überlegung auch nur ansatzweise umschrieben; auch ihr latenter Handlungsbezug wird

1 Frankfurt (1971), 11. 
nicht erläutert. Von daher kann sich die erste Stufe nicht anders als unterdeterminiert und ergänzungsbedürftig präsentieren. Denn praktische Rationalität ist nur dort möglich, wo Wünsche graduiert sind, die Überlegung der zweckrationalen Struktur folgt und auf die Bestimmung der zu vollziehenden Handlung ausgeht. Wären Wünsche erster Stufe wirklich so amorph, wie es hier erscheint, so müßte es ein Rätsel bleiben, wie gleichwohl auf der ersten Stufe Rationalität und Überlegung möglich sind.

\section{Irrelevanz der zweiten Stufe}

Es mag richtig sein, könnte nun cin Verteidiger der 7wei-Stufen-Theorie einwenden, daß die Wünsche erster Stufe, d.h. ihre präferentielle Ordnung, weitgehend festlegen, auf welchen konklusiven Wunsch sich die Volition zweiter Stufe zu richten habe; doch warum sollte es nicht trotzdem solche Volitionen geben?

Dieser Einwand gesteht zu, daß Volitionen zweiter Stufe im Falle rationalen Ilandelns zur Selektion bzw. Orienticrung des Handelns nichts beitragen. Ihre Stellungnahme ist demnach pro forma; sie stellen eine ,höhere" Instanz dar, die nichts zu melden hat. Dem oben erwähnten Zeiger, der auf einen der Wünsche erster Stufe deutet und ihn zur Effektivität designiert, wird von „,von unten“ souffliert, wo er hinzeigen muß. Oder um ein anderes Bild zu wählen, Volitionen sind wie konstitutionelle Monarchen, denen fertige Gcsetze zur Unterschrift vorgelegt werden, die aber keine gesetzesgestaltende Rolle spielen.

Bleibt die Frage, ob Volitionen, wenn sie schon keine inhaltlich selektive Funktionen haben, nicht doch in bezug aufs Handeln eine tragende kausale Rolle spielen. Auch ein konstitioneller Monarch spielt ja u.U. eine wichtige Rolle, indem das Gesetz nicht in Kraft tritt, solange er nicht unterschrieben hat. Diese Frage nach der kausalen Rolle scheint mit schwer entscheidbar zu sein, besonders vom Schreibtisch aus, weil sie auch eine empirische Seite hat. Einiges spricht aber doch begrifflich gesehen gegen die Annahme einer kausalen Rolle. Es scheint, daß in aller Regel die Gesetze - um im schon verwendeten Bild zu bleiben - auch ohne Unterschrift von oben in Kraft gesetzt und durchgesetzt werden; der König jedoch mit einer gestelzten Formulierung wichtigtuerisch seine Akzcptanz zun Ausdruck bringt, ohne damit der Geltung etwas hinzuzufügen.

Diese Einschätzung beruht darauf, daß eine Volition zweiter Stufe einem konklusiven Wünschen erster Stufe zum Verwechseln ähnlich sieht und die gleichen Wirkungen hat. Nehmen wir folgendes Beispiel: Meine praktische Überlegung hat ergeben, daß 3 ich am besten umgehend eine bestimmte Rechnung begleiche. Ich nehme mir daher vor, d.h. wünsche konklusiv, zur Bank zu gehen und das Geld zu überweisen. Wird dieser Wunsch kausal wirksam, so gehe ich zur Bank und veranlasse die Überweisung. Der konklusive Wunsch wird dadurch erfüllt und erweist sich als Wille, d.h. als effektiv.

Angenommen nun ich hätte jenen konklusiven Wunsch und schwinge mich außerdem - weil ich nicht bloß rational, sondern auch Person in Frankfurts Sinn sein möchte - zu der höherstufigen Volition auf, mein Wunsch, zur Bank zu gehen und die Überweisung zu machen, sei der effektive. Ich bin damit dann gewissermaßen doppelt entschlossen. Gehe ich nun zur Bank, so erfülle ich damit zunächst meinen konklusiven 
Wunsch erster Stufe, zugleich und mit ganz der gleichen Handlung auch die Volition zweiter Stufe. Denn diese ist ebenfalls genau dann erfüllt, wenn die Handlung vollzogen wird. Der Wunsch erster Stufe erweist sich dadurch als Wille und das war es ja, was die Volition auf zweiter Stufe zum Inhalt hatte. Von außen gesehen kann ein Beobachter nicht entscheiden, ob bei mir der einfache Wunsch, die Rechnung zu zahlen, wirksam war, oder aber der Wunsch, mein Wunsch, die Rechnung zu zahlen, möge effektiv sein. Auch ich selber aus der Perspektive des handelnden Subjekts hätte es schwer, die Wirksamkeit der beiden Wünsche auseinanderzuhalten. Denn wie ich es auch drehe und wende: Die Erfüllung und Wirksamkeit des einen wie des andern läuft auf genau dieselbe Handlung hinaus: daß ich zur Bank gehe und das Geld überweise.

Die Volition zweiter Stufe, daß der Wunsch, $h$ zu tun, effektiv ist und der konklusive Wunsch, h zu tun, erweisen sich damit in wesentlichen Hinsichten als äquivalent. Sie haben dieselben Erfüllungsbedingung und sie haben, wenn sie effektiv sind, dieselben Wirkungen, nämlich den Vollzug der Handlung h. Daraus schließe ich, daß die Volition zweiter Stufe nichts anderes ist, als eine geschraubte Art, einen konklusiven Wunsch erster Stufe auszudrücken.

\section{Irrationalität der zweiten Stufe}

Ich habe argumentiert, daß die zweite Stufe das Ergebnis der praktischen Überlegung erster Stufe aufzugreifen hat. Das läuft darauf hinaus, daß die zweite Stufe inhaltlich gesehen keine eigene Instanz darstellt. lch möchte nun kurz überprüfen, was sich ergeben würde, wenn im Gegensatz dazu die zweiten Stufe doch mit einer eigenen selektiven Funktion ausgestattet würde. Phänomenal gesehen entspräche dem der Fall, daß eine Person etwas aufgrund praktischer Überlegungen konklusiv wünscht und zugleich wünscht, dieser Wunsch sei nicht effektiv. Die Person würde sich qua höherer Instanz noch einmal gegen ihren auf die beste Handlung gerichteten konklusiven Wunsch erster Stufe bzw. dessen Effektivität aussprechen. Die zweite Stufe müßte dann einen anderen Wunsch dazu designieren, effektiv zu sein.

Sind solche Fälle möglich? Möglicherweise könnte man es so beschreiben, wenn eine Person die Haltung hat, immer irgendetwas anderes tun zu wollen, als sie rational wünscht. Dabei hätte man sich diese Einstellung als unbegründet, als einfach so vorhanden, zu denken, da die entsprechende Volition zweiter Stufe von den Wünschen erster Stufe unabhängig sein müßte. Wie auch immer man es sich im einzelnen vorstellt, es würde darauf hinauslaufen, daß die Person wünscht, nicht rational zu handeln.

Sind solche Fälle möglich? Sie sind sicher nicht unmöglich, wenn auch nicht leicht vorstellbar. Doch scheint es mir abwegig, gerade diese randständige und ambivalente Möglichkeit zum Charakteristikum von Person zu erklären. Personen würden sich demnach dadurch auszeichnen, daß sie sich auf zweiter Stufe dezisionistisch gegen ihre auf erster Stufe angesiedelte praktische Rationalität wenden können. Die Fähigkeit zur - auf zweiter Stufe - gewollten Irrationalität wäre das Kennzeichen von Personen - eine Lesart, die immerhin zu Frankfurts Feststellung paßt, ,that the essence of being a person lies not in reason but in will“. An dieser Stelle zeigt sich die Nähe der Zwei-Stufen- 
Theorie „persönlichen“ Handelns zur existentialistischen Idee grundlosen Entscheidens, wenn auch Frankfurt diesen Zusammenhang nicht explizit herstellt.

\section{Kurze Skizze einer Alternative}

Die bisher vorgetragenen Kritikpunkte laufen im Kern alle darauf hinaus, daß die ZweiStufen-Theorie der Dimension praktischer Begründung und Überlegung keinen Raum gibt. Sie arbeitet mit einer zu schwach strukturierten Sicht der Verhältnisse auf erster Stufe, ja sie gründet geradezu auf ihr. Daraus resultiert eine konzeptuelle Lücke. In diese treten dann Volitionen zweiter Stufe, ohne daß durch sie die Versäumnisse auf der ersten Stufe wirklich wettgemacht werden könnten. Das kann nicht gelingen, da auch die Volitionen zweiter Stufe nicht in eine Begründungsstruktur eingebettet sind; die zweite Stufe ist ebenso unterbestimmt wie die erste.

Dieser Befund spricht dafür, daß bereits die erste Stufe reicher strukturiert werden muß. Hier bietet sich natürlich die schon oben kurz angesprochene Struktur der praktischen Überlegung an, die von graduierten Wünschen erster Stufe ausgeht und mit einem konklusiven, handlungsbestimmenden Wünschen oder Wollen zum Abschluß kommt. In Falle rationalen Handelns werden diese Handlungsintentionen dann umgehend in die Tat umgesetzt. Handlungsbegründung und -erklärung spielen sich primär auf erster Stufe ab. Damit ein Wunsch wirksam wird und zum Handeln führt braucht es keines weiteren höherstufigen Wunsches, sondern ein Wunsch wird in der Regel dann wirksames Wollen, wenn er sich als Resultante einer praktischen Überlegung darstellt.

Nun hatte sich aber Frankfurts Zwei-Stufen-Theorie vor allem dadurch empfohlen, daß sie für die Phänomene irrationalen, unwillentlichen Handelns eine subtile Beschreibung vorlegt. Diese Phänomene beinhalten einen doppelten Willen, weil bei ihnen stets ein Wünschen und Wollen festzustellen ist, das zielstrebig seine Erfüllung erlangt; ebenso aber auch ein ineffektives Wünschen und Wollen da ist, das sich nicht erfüllt und dadurch zu einer Quelle von Frustration wird. Diese Ambivalenz deutet aber an sich nicht auf eine selbstbezügliche zweite Stufe des Wünschens oder Wollens. Dagegen springt es ins Auge, daß das eine Wünschen oder Wollen - das ineffektive - irgendwie vernünftig und überlegt ist, während das andere Wünschen oder Wollen sich wie hinterrücks durchsetzt. Um diese Doppelsinnigkeit des Willens zu artikulieren, braucht es zwar den Begriff eincs reflektierten im Sinne eines überlegten Willens, aber es braucht dazu' keinen in sich reflektierten, d. h. selbstbezüglichen Willen höherer Stufe.

Die Doppeldeutigkeit des Willens beim Phänomen unwillentlichen Handelns läßt sich rein auf der ersten Stufe artikulieren, wenn man zwischen einerseits überlegten evaluativen Wünschen, andererseits motivational wirksamen Wünschen unterscheidet. Lvaluative Wünsche sind präferentielle Einstellungen mit propositionalem Gehalt, die in der Regel bewußt sind. Auf der Grundlage solcher Wünsche wird die praktische Überlegung geführt, und sie sind auch die Zwischenergebnisse und Endpunkte dieser Überlegung in Form konklusiver Wünsche. Unter motivationalen Wünschen hingegen sind diejenigen Wünsche zu verstehen, die sich aus dem Verhalten einer Person er- 
schließen lassen. Normalerwcise, d.h. im Fall rationalen Handelns, sind die evaluativen Wünsche zugleich die motivierenden Wünsche. In spezicllen Fällen kann sich hier jedoch eine Differenz zeigen: Die Person handelt anders, als sie es evaluativ und überlegt will, wobei das abweichende Verhalten sich durchaus als wunschgesteuert präsentiert. Diese Wünsche sind die Motive des Handelns und lassen sich aus ihm erschließen. Diese motivationalen Wünsche sind nicht notwendig kongruent mit denen, von denen her die Person ihre praktische Überlegung führt.

Mit Hilfe dieser Unterscheidung lassen sich die Phänomene unwillentlichen Handelns als innere Inkongruenz der ersten Stufe fassen: Der widerwillig Drogensüchtige möchte evaluativ und überlegterweise die Droge nicht mehr nehmen, jedoch ist sein motivationaler Wunsch nach Drogenkonsum überproportional stark und setzt sich durch. Die beiden Wünsche unterscheiden sich dem Grade bzw. ihrer Stärke nach, weshalb es zur Diskrepanz kommt. Außerdem hat die Person zu diesen beiden Wünschen eine andere Zugangsweise: Die evaluativen sind ihr bewußt oder sie macht sie sich bewußt als subjektive Präskriptionen; dagegen sind die motivationalen Wünsche bzw. ihre Stärke Personen nicht unbedingt bekannt. Diese Wünsche befinden sich insofern „hinter dem Rücken“ der Handelnden, als sie sich letztlich erst im faktischen Verhalten der Person zeigen.

Es drängt sich die Frage auf, wieso es einen einschneidenden Unterschied macht, ob man die Phänomene unwillentlichen Handelns einstufig oder zweistufig beschreibt. Dazu muß man sich klar machen, daß diese Fälle bei Frankfurt dazu dienen, den ZweiStufen-Ansatz erstmals phänomenal zu validieren, um von da aus dann zu einer Beschreibung voll willentlichen Handeln fortzuschreiten. Unwillentliches und willentliches Handeln stellen sich natürlicherweise als komplementär zueinander dar. Das hat bei Frankfurt zur Konsequenz, daß willentliches Handeln ebenfalls unter das Dach der Zwei-Stufen-Theorie gebracht wird. Die zweistufige Auffassung unwillentlichen Handelns ist somit ein entscheidender Schritt für die Plausibilisierung einer zweistufigen Konzeption willentlichen Handelns.

Bei willentlichem Handeln soll jeweils eine Volition zweiter Stufe nicht bloß vorhanden, sondern auch wirksam sein: Sie verwandelt - gleich einem Zauberstab - einen bloßen Wunsch erster Stufe in einen Willen und führt damit indirekt zu entsprechendem Handeln. Willentliches Handeln verdankt sich also jeweils einer „durchschlagenden” Volition zweiter Stufe. ${ }^{2}$ Wenn man davon ausgeht, daß willentliches Handeln den Normalfall rationalen, überlegten Handelns darstellt, so müßten durchschlagende Volitionen sehr häufig auftreten. Gleichzeitig handelt es sich aber um höchst eigenartige Wünsche, um Wünsche nämlich, die die Kraft haben, sich selbst zu erfüllen. Diese Wünsche sollen durch bloße Kraft des Wünschens dort ein Wollen erzeugen, wo ohne sie keines wäre. Doch es fragt sich, wie eine Person auf höherer Stufe die „Wunschkraft“ erlangen kann, bei sich selber die Wirksamkeit von Wünschen zu verursachen, wenn es andererseits derselben Person nicht möglich ist, ihre Wünsche erster Stufe in die Tat umzuset-

1 Den Unterschied sowie die rationale Identität von Evaluation und Motivation habe ich näher ausgeführt in Kusser (1989), Teil III.

2 Die damit verbundene Vorstellung eines sich selbst erfüllenden Wunsche ist in der Literatur verschiedentlich kritisiert worden. Vgl. Baumann (2000) Kap. 5.2. 
zen. Diese Vorstellung erinnert ein wenig an Baron Münchhausens Geschichte, der sich selbst aus dem Sumpf gezogen haben will.

Der wichtigste Unterschied zwischen Zwei-Stufen-Theorie und einstufigem Ansatz liegt in der Konzeption des rationalen Handelns. Während die Zwei-Stufen-Theorie für jede Episode rationalen Handelns ein Wunder "von oben" postuliert, kommt es nach dem einstufigen Ansatz immer dann zu willentlich rationalem Handeln, wenn der präferentielle Rang eines Wunsches seiner motivationalen Kraft entspricht. Eine solche Kongruenz ist der Normalfall, so daß man in der Regel davon ausgehen kann, daß die evaluativen, einstufigen Wünsche einer Person direkt zu wunschverwirklichendem Handeln führen. Man muß also beim rationalen Handeln keinen zusätzlichen (,höherstufigen“) Kausalfaktor ansetzen, sondern es ist umgekehrt im Falle unwillentlichen Handelns ein zusätzlicher Kausalfaktor im Spiel. Dieser stört dann die normale Kausalität der Evaluation, so daß eine diskrepante Motivation resultiert, die in inkongruentes Verhalten mündet.

Verglichen damit kompliziert die Zwei-Stufen-Theorie die Genese willentlichen Handelns unnötig. In diesen Fällen ist die für unwillentliches Handeln charakteristische Willensambivalenz nicht gegeben. Obwohl damit der Grund entfällt, mit einem „doppelten" Willensbegriff zu arbeiten, wird er zweistufig konzipiert, da die Zwei-StufenTheorie diesen Fall unzweideutigen Wollens von seinem anormalen Gegenstück her aufrollt.

Aus meiner Sicht kann und sollte die Grundkonzeption normalen, rationalen Handelns von zweistufigen Wünschen freigehalten werden. Das bedeutet nicht, daß es Wünsche zweiter Stufe generell nicht geben könnte oder nicht gäbe. Im Gegenteil gibt es eine Reihe von speziellen Phänomenen, bei denen Wünsche zweiter Stufe eine Rolle spielen. So ist etwa eine Volition zweiter Stufe die naheliegende Reaktion auf die Erfahrung eigenen unwillentlichen Handelns. Durch diese Volition wird gewünscht, evaluative und handlungswirksame motivationale Wünsche seien im Einklang. Vermutlich wird der unwillentlich Drogensüchtige immer wieder wünschen, seine Motivation zum Drogenkonsum sei nicht so stark und könnte in Schach gehalten werden von seinem evaluativen Wunsch nach einem drogenfreien Leben. Er möchte - und dies ist tatsächlich ein Wunsch zweiter Stufe - in einen Stand kommen, in dem seine evaluativen Wünsche die Handlung ,regieren.” Um das zu erreichen, müßte er sich ernsthaft überlegen, wie sich seine Motivation in entsprechende Form bringen ließe, wozu es beispielsweise auch gehören könnte, motivational störende Einflüsse (wie körperliche Entzugserscheinungen) zu dämpfen; es bliebe ihm nicht erspart, auf die Handlungsebene herunterzusteigen, um diesen höherstufigen Wunsch zu verwirklichen. Es würde also gerade nicht darum gehen, diesen Wunsch direkt innerpsychisch in einen Stand zu versetzen, wo er sich selbst verwirklicht, sondern es ginge darum, diesen Wunsch - wie andere Wünsche auch - zu verwirklichen, indem zu zielführendem Handeln geschritten würde.

Nach diesem Szenarium ist der Wunsch zweiter Stufe keine tragende Säule der $B e$ schreibung unwillentlichen Handelns, sondern eine zukunftsbezogene Reaktion darauf. Außerdem läßt sich dieser Wunsch bewußtseinsmäßig greifen, er fließt in eine praktische Überlegung ein und führt in der Regel zu entsprechenden Handlungen. Die Rolle von Wünschen zweiter Stufe ist also insgesamt eine andere als in Frankfurts Zwei- 
Stufen-Theorie. Sie treten erst im Gefolge defizitären Handelns auf, spielen jedoch keine tragende Rolle in der Beschreibung und Erklärung willentlichen und unwillentlichen Handelns. Dadurch kann der praktischen Überlegung ihr angestammter Platz in der Genese menschlichen Handelns erhalten bleiben.

\section{Literatur:}

Baumann, Peter (2000), Die Autonomie der Person, Paderborn

Frankfurt, Harry G. (1971), Freedom of the Will and the Concept of a Person, Journal of Philosophy, Vol. LXVIII, 5-20

Gosepath, Stefan (1992), Aufgeklärtes Eigeninteresse, Frankfurt/M.

Kusser, Anna (1989), Dimensionen der Kritik von Wünschen, Frankfurt/M.

Nunner-Winkler, Gertrud. (1993), Die Entwicklung moralischer Motivation, in: Moral und Person, hg. v. Wolfgang Edelstein, Gertrud Nunner-Winkler, Gil Noam, Frankfurt/M.

Nunner-Winkler, G. (2000), Von Selbstzwängen zur Selbstbindung, in: Anthropologie und Moral, hg. v. Martin Endreß, Würzburg

Wolf, Susan, (1990), Freedom within Reason, Oxford 